\title{
Loss Tolerance in One-Way Quantum Computation via Counterfactual Error Correction
}

\author{
Michael Varnava, ${ }^{1}$ Daniel E. Browne, ${ }^{2}$ and Terry Rudolph ${ }^{1,3}$ \\ ${ }^{1}$ QOLS, Blackett Laboratory, Imperial College London, Prince Consort Road, London SW7 2BW, United Kingdom \\ ${ }^{2}$ Department of Materials and Department of Physics, University of Oxford, Parks Road, Oxford, OX1 3PU, United Kingdom \\ ${ }^{3}$ Institute for Mathematical Sciences, Imperial College London, 53 Exhibition Road, London SW7 2BW, United Kingdom
}

(Received 18 May 2006; published 20 September 2006)

\begin{abstract}
We introduce a scheme for fault tolerantly dealing with losses (or other "leakage" errors) in cluster state computation that can tolerate up to $50 \%$ qubit loss. This is achieved passively using an adaptive strategy of measurement-no coherent measurements or coherent correction is required. Since the scheme relies on inferring information about what would have been the outcome of a measurement had one been able to carry it out, we call this counterfactual error correction.
\end{abstract}

PACS numbers: 03.67.Lx, 03.67.Pp

Quantum computation architectures can only be considered viable if they are demonstrably fault tolerant. Thresholds on fault tolerance are typically quoted as around $0.01 \%$ - that is, the error on a generic gate operation or state preparation should be less than this amount. However, one hopes that for specific error models well chosen strategies might significantly relax this rather stringent requirement [1].

A new architecture for quantum computation that is generating much interest is the cluster state model [2-4], otherwise known as "one-way quantum computation." In this approach, the quantum computation proceeds in two stages. First, a special kind of entangled multiparty state, called a cluster state, is generated. The quantum computation is then implemented by single qubit measurements; the specific algorithm computed is a function of the choice of measurement bases, and the order in which they are made. There are two main approaches to tackling fault tolerance in a new architecture. One is to translate standard fault tolerance techniques to the new model, an approach which has been successful for the cluster state quantum computation [5-7]. A different approach is to develop novel protocols which exploit the features of the architecture, in this case the entanglement of the cluster states themselves. This is the approach we have adopted here. A further recent development in this vein is a fault-tolerant model which exploits the fact that certain cluster states are topological error correcting codes [8].

A main result of this Letter will pertain to architectures which can produce and utilize cluster states in such a way that a significant error mechanism involves each qubit in the cluster being lost (or undergoing some other sort of detectable failure) with fixed and independent probability $\varepsilon_{0}$. What we will show is if $\varepsilon_{0}<50 \%$ then computation fault tolerant to loss errors is possible. Remarkably, this can be achieved with only destructive measurements, and without coherent correction mechanisms needing to be applied to the qubits.

We begin by introducing cluster state structures for quantum computation which are naturally resilient to qubit loss. To simplify the first part of our discussion, we shall initially assume that any loss errors occur after the entangled state has been generated. Later on, we shall relax this requirement.

We use $X_{i}, Y_{i}, Z_{i}$ to denote the Pauli operators on qubit $i$, and define cluster states in terms of their stabilizers [3] as follows: A cluster state is represented by a graph, where the $n$ vertices of the graph denote qubits, while the bonds denote a certain entangling operation between them. Denoting by $E(i)$ the set of edges on this underlying graph which are connected to vertex $i$, the cluster state is that state which is invariant under the action of the $n$ stabilizer operators: $X_{i} \prod_{j \in E(i)} Z_{j}$.

Equivalently, the cluster state is the state obtained by preparing each qubit in the state $|0\rangle+|1\rangle$ and then applying a CPHASE (controlled phase-flip) gate to every pair of qubits connected by an edge on the graph.

Since the cluster states are eigenstates of the stabilizers, they predict with certainty correlations in the measurement outcomes of certain sets of measurements. To illustrate this, consider a two-qubit state stabilized by the operator $X_{1} Z_{2}$. If observable $X_{1}$ is then measured the outcome of $Z_{2}$ is now known with certainty. We say that the measurement $X_{1}$ is an indirect measurement of the observable $Z_{2}$. Importantly, if such a state is prepared, and qubit 2 undergoes a loss error, $Z_{2}$ can still be measured indirectly, even though qubit 2 is no longer available. The principle of applying counterfactual reasoning to the correlations in cluster states to effect indirect measurements on lost qubits, lies at the heart of our scheme.

The cluster state structure we utilize is that of a tree (see Fig. 1), which can be specified by its branching parameters $b_{i}$ as indicated. The tree graphs are designed in this way to take full advantage of the three general cluster state properties summarized in Fig. 2. In particular, a crucial feature of these tree clusters is that any given qubit within the cluster can be removed indirectly (utilizing an effective $Z$ measurement) by performing measurements on a subset of the qubits below it in the cluster. This is depicted in Fig. 3. 


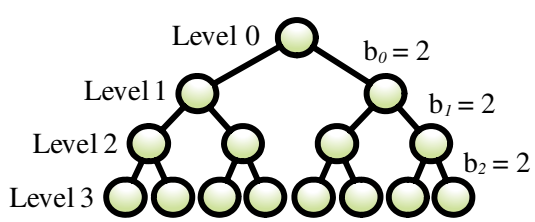

FIG. 1 (color online). A tree cluster with branching parameters $b_{0}=b_{1}=b_{2}=2$.

Suppose we desire to perform a quantum computation using the cluster shown in Fig. 4(a). In general, the computation requires the measurement of some observable, $A(\alpha) \equiv \cos \alpha X+\sin \alpha Y$, directly on a given qubit as indicated. However, due to the properties (b) and (c) of Fig. 2, it is clear that an equivalent measurement procedure is given by Fig. 4(b). The important point to note, is that there is an alternative measurement pattern-that of Fig. 4(c) - which also achieves exactly the same effect. Now, since they are in the Pauli group, the pair of $X$ measurements can be performed "offline," i.e., as part of preparing the cluster state for the computation and prior to the computation having reached this position. Alternatively the cluster state which results after these $X$ measurements could be grown directly.

The loss tolerance of this proposal stems from the following observation. If the $A$ measurement attempted in Fig. 4(b) fails to register an outcome due, for example, to a loss error, then we try to remove this qubit from the cluster by an indirect $Z$ measurement, and proceed to make the $A$ measurement as in Fig. 4(c). If this measurement also fails (or if any of the necessary $Z$ measurements fail) then the computation has failed. As we now show, the generalization of this simple procedure to a tree with larger branching parameters can greatly reduce the effective qubit loss rate and lead to a successful implementation of the $A$ measurement.

a)

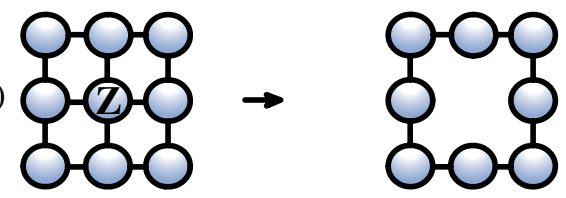

b)

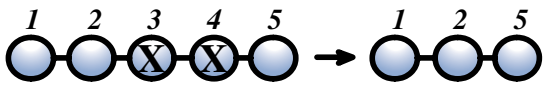

c)

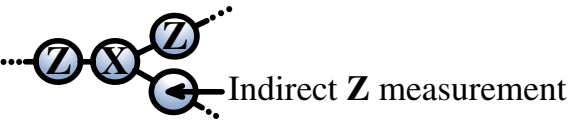

FIG. 2 (color online). Certain measurements on a graph qubit leave the remaining qubits in a new cluster state with a different layout: (a) A $Z$ eigenbasis measurement removes the qubit from the cluster and breaks all bonds between that qubit and the rest. (b) Two adjacent $X$ measurements on a linear cluster remove the qubits and form direct bonds between their neighbors. (c) Measuring a qubit in the $X$ basis and performing $Z$ measurements on all but one of the remaining directly bonded qubits deterministically reveals what the $Z$ measurement outcome would have been on the unmeasured qubit.

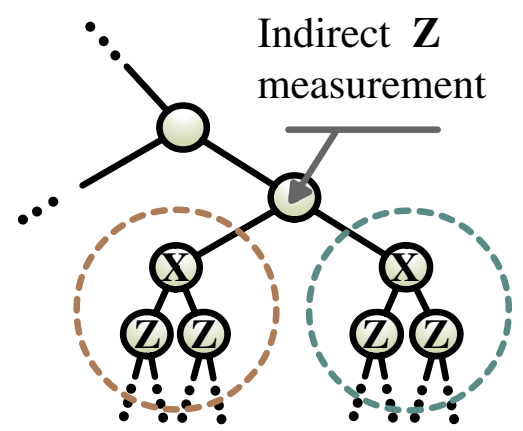

FIG. 3 (color online). An indirect $Z$ measurement will be performed on the indicated qubit if either the three measurements circled in red are successful, or if those circled in green are successful.

Note that the order of the measurements demanded by the our loss-tolerance protocol (as described in the previous paragraph) is not the one that would allow the implementation of a deterministic rotation gate. In fact, the sign of this rotation angle depends on the outcome of measurements which must (for loss tolerance) be made after $A$ itself is measured. Thus with equal probability a rotation corresponding to either $A(\alpha)$ or $A(-\alpha)$ occurs. Fortunately, by adopting strategies described in Ref. [9] this effect can be overcome at the cost of a small overhead in the size of the computation. Note that the measurement order imposed in the equivalent one-way quantum computation without loss tolerant encoding (as described in the introductory paragraphs) must still be respected, as otherwise one could not determine whether $A(\alpha)$ or $A(-\alpha)$ had

a)

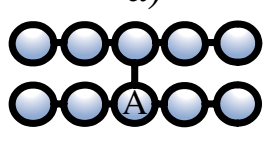

Equivalent to:

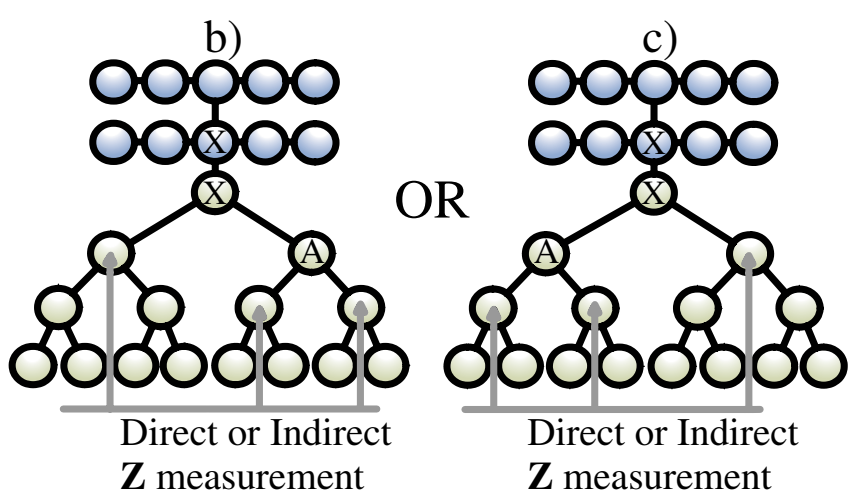

FIG. 4 (color online). A standard cluster computation requires measurement of observable $A$ on the indicated qubit, as indicated in (a). Because of the properties depicted in Fig. 2, either of the two measurement patterns depicted in (b) and (c) will implement the desired measurement of $A$. 
been implemented and thus which corresponding correction strategy one should employ.

Consider a tree cluster with branching parameters $b_{0}, b_{1}, b_{2}, \ldots, b_{m}$. The probability of successfully performing the necessary measurement pattern to implement the $A$ measurement on such a tree is given by

$$
P=\left[\left(1-\varepsilon_{0}+\varepsilon_{0} R_{1}\right)^{b_{0}}-\left(\varepsilon_{0} R_{1}\right)^{b_{0}}\right]\left(1-\varepsilon_{0}+\varepsilon_{0} R_{2}\right)^{b_{1}},
$$

where for $k \leq m$

$$
R_{k}=1-\left[1-\left(1-\varepsilon_{0}\right)\left(1-\varepsilon_{0}+\varepsilon_{0} R_{k+2}\right)^{b_{k+1}}\right]^{b_{k}}
$$

and $R_{m+1} \equiv 0, b_{m+1} \equiv 0$.

The $R_{i}$ are defined as the probabilities of successfully implementing an indirect $Z$ measurement on any given qubit found in the $i$ th level. Thus $\left(1-\varepsilon_{0}\right)+\varepsilon_{0} R_{i}$ is the probability of a successful $Z$ measurement on a qubit at level $i$. The Eqs. (2) can be derived recursively, starting from the qubits at the bottom of the tree.

The derivation of $P$ can be understood as follows. Imagine one proceeds along the $b_{0}$ qubits until the $A$ measurement succeeds on qubit number $k$. Overall success, given that the $A$ measurement has succeeded on qubit $k$, requires: (i) Successful (direct or indirect) $Z$ measurements on the $b_{1}$ qubits below qubit $k$, (ii) indirect $Z$ measurements to succeed on the $k-1$ qubits that the $A$ measurement failed on and (iii) direct or indirect $Z$ measurements to succeed on the remaining $b_{0}-k$ qubits. This leads to an overall success probability $P$.

Let us introduce the parameter $\varepsilon_{\text {eff }} \equiv 1-P$ to represent the overall effective loss rate for the tree-encoded logical cluster qubit. For a range of fixed $\varepsilon_{0}$ and $\varepsilon_{\text {eff }}$ we have performed a numerical search over integer values for the branching parameters $b_{0}, b_{1}, \ldots, b_{m}$ which minimize the number of qubits $Q$ required in the tree. These results are depicted in Fig. 5. (Note the plot is log versus loglog.) We have shown the abscissa to somewhat extreme values only to indicate the important scaling. The linear nature of the plot implies that $Q=\operatorname{polylog}\left(1 / \varepsilon_{\text {eff }}\right)$, which demonstrates that this procedure has the scaling properties required for proper fault tolerance.

When $\varepsilon_{0} \geq 0.5$, numerical simulations show that $\varepsilon_{\text {eff }}$ cannot be lowered from $\epsilon_{0}$. This indicates a loss-tolerance threshold of 50\%. This can be understood as follows. If the above-described scheme could correct loss errors with an error rate at $50 \%$ or higher, this would violate the nocloning theorem [10]. To see this, consider a 4-qubit linear cluster state. Alice is given the first three qubits, and Bob is given the last, which Bob then immediately encodes in a tree. Alice makes measurements with bases unknown to Bob. Now, imagine a third party, Charlie, steals $50 \%$ of Bob's qubits, chosen at random. This would be indistinguishable (to Bob) from the qubits having a 50\% loss rate. If our protocol (with a nondemolition measurement replacing the measurement in basis " $A$ ") allowed this level of

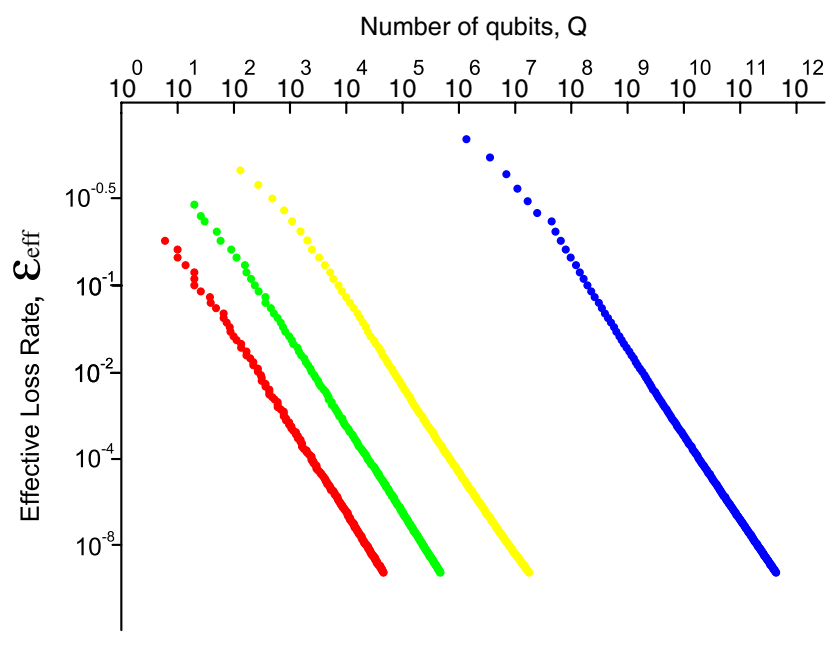

FIG. 5 (color online). Numerical results showing the number of qubits required versus the desired effective loss rate. The different curves, from left to right, correspond to $\varepsilon_{0}=0.2,0.3$, $0.4,0.49$, respectively.

loss to be tolerated, both Bob and Charlie could produce "clones" of the state prepared by Alice's measurements violating the no-cloning theorem.

Interestingly, by allowing an adaptive construction of the tree based on which particular qubits in the first row are lost, the no-cloning theorem is no longer an obstacle and a strategy can be found for arbitrarily high loss rates [11]. However, this strategy needs deterministic gate operations to adaptively build up the tree, which makes it incompatible with nondeterministic cluster state generation schemes [12-15].

So far, our error model has only explicitly considered loss errors occurring after the cluster state has been generated. Fortunately, we can relax that requirement in a number of practically relevant scenarios. First let us consider cluster state generation via deterministic CPHASE gates. Loss errors can occur before the CPHASE without disturbing the efficacy of the loss-tolerance protocol if the attempted physical entangling operation acts as the identity (up to a global phase) when only one physical qubit carrier is present. The loss error takes the state outside of the computational basis and so the action of an abstract CPHASE gate is not defined. However, for any physical implementation of such an operation, this can be assessed and, indeed, a number of proposed quantum gates, e.g., [16], satisfy this criterion.

To confirm the loss correction protocol also succeeds in this case, recall that obtaining the +1 outcome of a $Z$ measurement on a given qubit in the cluster leaves the remaining qubits in a cluster state with the same graph, but with all edges attached to the measured qubit removed (as illustrated in Fig. 2(a). This, however, is the same state which would have been obtained if no entangling operations on that qubit had been applied or, equivalently, if that qubit had suffered a loss error before the operations. Thus in this case loss events before the entangling operation are 
equivalent to loss events afterward, and will be corrected in the same way.

In linear optical quantum computation, deterministic CPHASE gates are not available. It is possible to combine nondeterministic gates with a near-deterministic gate teleportation approach [17], or, more efficiently, generate the cluster state via nondeterministic gates and an appropriate strategy [12-14]. However, the most resource efficient approach is to employ so-called nondeterministic "fusion operations" [13] via polarizing beam splitter networks. This setup requires a more complicated analysis, but in this case also, loss errors occurring before and during the beam splitter network can still be tolerated.

In practice, of course, a quantum computation must be tolerant to more than loss errors. In this regard there are several important things to note about our scheme. First, because of the large overhead in numbers of qubits in the tree, one might naively expect that the loss tolerance comes at the expense of creating large sensitivity to depolarizing noise. In fact, this is not the case. For a start, when increasing the size of the tree, the vast majority of the extra qubits will only ever be measured in a Pauli basis. For example, for an initial loss rate $\varepsilon_{0}=0.2$, to get $\varepsilon_{\text {eff }}=$ $10^{-10}$ then non-Pauli measurements will only be attempted on at most 15 of the qubits forming each tree. Note also that the vast majority of operations here are in the Clifford group. Robust fault-tolerant methods of implementing Clifford operations are well-known (see, e.g., Ref. [18]).

However, additional encoding of these operations is not even necessary. There is, in fact, a natural robustness against general errors in our loss-tolerance protocol. This is because the tree structure leads to a great deal of redundancy. For every qubit, there are a number of different ways an indirect measurement can be made, e.g., measurements on different subsets of qubits below it on the tree. These different sets of measurements would, in the ideal case, all lead to the same result, but the presence of additional noise will lead to errors. Erroneous indirect measurements will lead to additional logical errors (both local depolarization and non-Markovian errors [5,7]) in the computation. Adopting a simple majority voting strategy, however, significantly decreases the overall error rate. In fact, the greater the branching ratios in the trees, the lower the probability of an incorrect majority vote.

Qubits and measurements in the first row of the tree (the qubits which may need to be measured in a non-Pauli basis) are not protected from errors (and the negligible residual error caused by incorrect indirect measurements) by such a strategy. However, by encoding these qubits further, using an error-detecting code, more general errors could be detected and located. The protocol can tolerate located errors in the same way as losses. The 15-qubit quantum Reed-Muller code [19] is most suitable for this task [8] as it is the only code known so far, where the nonPauli measurements on the encoded qubit can be achieved with single qubit measurements.
Our primary purpose in this Letter has been to show that an extremely high error threshold exists for one of the primary error mechanisms affecting several quantum computational architectures based on the one-way model. This is achieved using treelike cluster state structures to implement counterfactual measurements on qubits which have undergone a loss error. Our scheme will be relevant to all realizations of cluster state quantum computation where qubit loss is an important source of error, especially optical implementations.

We acknowledge useful conversations with C.M. Dawson, C. Moura Alves, J. Franson, T. Jennewein, P. Kok, P. Kwiat, M. A. Nielsen, H. Ollivier, J.-W. Pan, T. Stace, I. Walmsley, P. Walther, and G. Weihs. This research was supported by the DTO-funded U.S. Army Research Office Contract No. W911NF-05-0397, The British Council, Merton College, Oxford, the QIPIRC, and the Engineering and Physical Sciences Research Council.

[1] E. Knill, Nature (London) 434, 39 (2005); M. Silva, M. Rötteler, and C. Zalka, Phys. Rev. A 72, 032307 (2005).

[2] R. Raussendorf and H. J. Briegel, Phys. Rev. Lett. 86, 5188 (2001).

[3] R. Raussendorf, D.E. Browne, and H. J. Briegel, Phys. Rev. A 68, 022312 (2003).

[4] D. E. Browne and H. J. Briegel, quant-ph/0603226.

[5] M. A. Nielsen and C.M. Dawson, Phys. Rev. A 71, 042323 (2005); C. M. Dawson, H. L. Haselgrove, and M. A. Nielsen, Phys. Rev. Lett. 96, 020501 (2006).

[6] R. Raussendorf, Ph.D. thesis, Ludwig-Maximilians University, Munich, 2003, online at http://edoc.ub. unimuenchen.de/archive/00001367/.

[7] P. Aliferis and D. W. Leung, Phys. Rev. A 73, 032308 (2006).

[8] R. Raussendorf, J. Harrington, and K. Goyal, quant-ph/ 0510135 .

[9] M. A. Nielsen, Phys. Lett. A 308, 96 (2003).

[10] W. K. Wooters and W. H. Zurek, Nature (London) 299, 802 (1982); D. Diekes, Phys. Lett. A 92, 271 (1982).

[11] T. Stace (private communication).

[12] M. A. Nielsen, Phys. Rev. Lett. 93, 040503 (2004).

[13] D. E. Browne and T. Rudolph, Phys. Rev. Lett. 95, 010501 (2005).

[14] S. D. Barrett and P. Kok, Phys. Rev. A 71, 060310(R) (2005).

[15] K. Kieling, D. Gross, and J. Eisert, quant-ph/0601190; D. Gross, K. Kieling, and J. Eisert, quant-ph/0605014 [Phys. Rev. A (to be published)].

[16] D. Jaksch, H. J. Briegel, J. I. Cirac, C. W. Gardiner, and P. Zoller, Phys. Rev. Lett. 82, 1975 (1999).

[17] E. Knill, R. Laflamme, and G. Milburn, Nature (London) 409, 46 (2001).

[18] M. A. Nielsen and I. Chuang, Quantum Computation and Quantum Information (Cambridge University Press, Cambridge, 2000).

[19] S. Bravyi and A. Kitaev, Phys. Rev. A 71, 022316 (2005). 